\title{
COMPOSTOS FENÓLICOS, CAROTENOIDES E ATIVIDADE ANTIOXIDANTE EM FRUTOS DE CAJÁ-MANGA
}

\author{
JOSÉ ROBERTO CHAVES NETO1 \\ MARIA DAS GRAÇAS DOS SANTOS ANDRADE ${ }^{2}$ \\ ANA PAULA PEREIRA SCHUNEMANN ${ }^{3}$ \\ SILVANDA DE MELO SILVA ${ }^{4}$
}

O presente trabalho teve como objetivo quantificar os compostos fenólicos, carotenoides e atividade antioxidante de frutos de cajá-mangueira de diferentes acessosde ocorrência espontânea no Estado da Paraíba. O delineamento experimental utilizado foi 0 inteiramente casualizado (DIC), com 3 acessos (A1, A2 e A3), em três repetições. Os frutos foram analisados quanto ao conteúdo de ácido ascórbico, carotenoides totais, flavonoides amarelos, antocianinas, polifenóis extraíveis totais e atividade antioxidante. Os acessos diferiram significativamente quanto ao conteúdo de ácido ascórbico, carotenoides totais, flavonoides amarelos e antocianinas. Os resultados demonstraram que o cajá-manga possui quantidades significativas de ácido ascórbico (46,49 mg.100 $\left.\mathrm{g}^{-1}\right)$. Considerando os baixos teores de flavonoides amarelos e antocianinas em polpa de cajá-manga, é possível inferir que a ação antioxidante exibida decorra, principalmente, da presença de ácido ascórbico e polifenóis extraíveis totais. O conteúdo de ácido ascórbico foi superior ao de frutas usualmente consumidas e atividade antioxidante próxima ao de frutas com um potencial antioxidante conhecido. A atividade antioxidante foi fortemente correlacionada com o conteúdo de ácido ascórbico. O conteúdo de ácido ascórbico, carotenoides totais, flavonoides amarelos e antocianinas contribuirão para a variabilidade entre os acessos, com ácido ascórbico inferindo a maior contribuição para esta variação.

PALAVRAS-CHAVE: PÓS-COLHEITA; FRUTOS TROPICAIS; ANADARDIACEAE; SPONDIASDULCISPARKINSON; ÁCIDO ASCÓRBICO; ABTS

\footnotetext{
'Engenheiro Agrônomo, Doutorando em Engenharia Agrícola - Universidade Federal de Santa Maria (UFSM), Santa Maria, RS, Brasil. (e-mail: jose.chavesneto@gmail.com). \# Autor para correspondência.

²Engenheira Agrônoma, Areia, PB, Brasil. (e-mail: mgandrade@hotmail.com).

3Doutora em Agronomia, Florianópolis, SC, Brasil. (e-mail: anaschunemann@gmail.com).

${ }^{4}$ Professora Adjunta no Departamento de Ciências Fundamentais e Sociais, Universidade Federal da Paraíba (UFPB), Areia, PB, Brasil. (silvandasilva@gmail.com).
} 


\section{INTRODUÇÃO}

Pertencente ao gênero Spondias e a família Anadardiaceae a cajá-mangueira (Spondias dulcis Parkinson) é uma frutífera exótica no Brasil ainda em fase de domesticação, originária da região da Indo-Malásia ao Taiti e encontrada em todas as regiões tropicais (Janick \& Paull, 2008). Segundo Franquin e colaboradores (2005) está espécie é principalmente cultivada no Caribe, Central e do Sul e na Ásia. No Caribe esta espécie é principalmente cultivada como árvores de terreiro; porém, já vem sendo consorciada com outras frutíferas de importância mundial como a banana, o cacau e algumas espécies cítricas.

A cajá mangueira é uma espécie distribuída espontaneamente na zona da mata, no agreste, nas regiões semi-áridas, sub-úmida e semi-úmida do nordeste brasileiro. Assim como outras espécies do gênero Spondias, tem no extrativismo sua principal forma de exploração (Sousa et al., 1998; Lorenzi et al., 2006). No Estado da Paraíba, é encontrada na mesorregião do Brejo Paraibano e Mata Paraibana. Porém, segundo Silva et al., (2012), é dispersa em um número reduzido de plantas em pomares domésticos e seus frutos são apreciados pela população local exclusivamente para consumo in natura.

No entanto, as espécies do gênero Spondias, assim como outras espécies frutíferas nativas e exóticas não tradicionais, da região Nordeste do Brasil são consideradas frutas "potenciais", uma vez que, apresentam características atrativas a agroindústria, e principalmente são tidas como uma fonte promissora de uma grande variedade de compostos bioativos reconhecidos por proporcionar benefícios à saúde (Silva et al., 2012), bem como pelo elevado consumo e aceitação pela população das áreas de ocorrência durante as safras (Moura et al., 2013).

O cajá-manga é caracterizado como drupa elipsoides, com sementes dotadas de fibras rígidas e espinescentes que mergulham parcialmente na polpa. Tido como um fruto suculento, agridoce e fortemente aromático. Sendo destinado em sua totalidade para 0 consumo in natura, devido às excelentes características organolépticas, rico em fibras e em sais minerais (Lorenzi et al., 2006). Um aspecto importante sob a qualidade destes frutos se dá principalmente devido ao teor de ácido ascórbico, pois segundo Dantas et al. (2011), os frutos de cajá-mangueira apresentam conteúdos variando de 73,12 a 81,06 mg.100g-1, sendo superiores aos de frutos de umbuzeiro (Spondiastuberosa Arruda) por Gonçalves et al. (2010) com valores médios de $52,98 \mathrm{mg} .100 \mathrm{~g}^{-1}$ de ácido ascórbico.

Juntamente com as características físicos dos frutos referentes à aparência externa, tamanho, forma e cor da casca, que dão a estes frutos sabor exótico os caracteres físicoquímicas relacionados ao sabor, odor, textura e principalmente valor nutritivo e conteúdo de compostos bioativos (conteúdo de ácido ascórbico), caracterizam excelente atributos de qualidade que permitem maior aceitação destes no mercado de frutos frescos e/ou pela indústria como matéria-prima no preparo de sucos, picolés, sorvetes, néctares, geleias, e principalmente de polpa congelada, devido a esses aspectos os frutos in natura têm participação crescente no agronegócio da região Nordeste (Carvalho et al., 2008; Aroucha et al., 2012).

Os compostos bioativos estão naturalmente presentes em frutas, ervas e hortaliças, por exemplo o ácido ascórbico, ácidos fenólicos e carotenoides (Ali et al., 2008; Lu et al., 2011). Estes compostos são responsáveis pela capacidade antioxidante, podendo atuar no organismo de modo a capturar os radicais livres produzidos em excesso que ocasionam a 
degeneração dos tecidos, podendo causar também doenças cardiovasculares, câncer, entre outras (Paliyath et al., 2008).

Associado a possível contribuição do cajá-manga como fonte promissora de compostos bioativos e atividade antioxidante, a escassez de trabalhos sobre o assunto evidencia a necessidade de mais investigações sobre a perspectiva dos frutos desta espécie serem uma fonte de compostos com elevado potencial antioxidante. Sendo assim, este trabalho teve o objetivo quantificar os compostos fenólicos, carotenoides e atividade antioxidante de frutos de cajá-mangueira de diferentes plantas de ocorrência espontânea no Estado da Paraíba.

\section{MATERIAL E MÉTODOS}

\subsection{PROCEDÊNCIA DOS FRUTOS E COLHEITA}

Os frutos foram colhidos manualmente pela manhã, no estádio de maturação maduro, caracterizado pela coloração da casca totalmente amarela, em três plantas (acessos) localizadas em diferentes municípios do Estado da Paraíba, A1 (Solânea), A2 (Cuité de Mamanguape) e A3 (João Pessoa). Após a colheita os frutos foram transportados em caixas térmicas com gelo, ao Laboratório de Biologia e Tecnologia de Pós Colheita, Centro de Ciências Agrárias (CCA), Universidade Federal da Paraíba (UFPB). No laboratório os frutos foram lavados e selecionados, descartando-se aqueles que apresentavam danos físicos ocasionados pelo manuseio e transporte, pelo ataque de doença ou praga, em seguida, foram preparadas 3 repetições de cerca de $250 \mathrm{~g}$ de polpa, homogeneizada de cada planta e armazenadas em freezer à temperatura de $-80^{\circ} \mathrm{C}$ para as determinações de:

\subsection{DETERMINAÇÃO DE COMPOSTOS BIOATIVOS}

\subsubsection{Teor de ácido ascórbico (mg.100 g-1)}

Para a quantificação do ácido ascórbico (mg.100 $\left.\mathrm{g}^{-1}\right)$, homogeneizou-se $1 \mathrm{~g}$ de amostra em $50 \mathrm{~mL}$ de solução de ácido oxálico $0,5 \%$ e titulada com a solução DFI (2,6 diclorofenol-indofenol $0,002 \%$ ), até a obtenção da coloração rósea claro permanente, sendo o ponto de viragem detectado visualmente, conforme Strohecker e Henning (1967).

\subsubsection{Carotenoides Totais ( $\mu \mathrm{g} .100 \mathrm{~g}-1)$}

Determinado por espectrofotometria a $450 \mathrm{~nm}$, conforme Higby (1962), utilizando $5 \mathrm{~g}$ de polpa para $10 \mathrm{~mL}$ da solução extratora de Hexano PA. Para cálculos foi utilizada a fórmula 1: Carotenóides totais $=(A \times 100) /(250 \times L \times W)$, onde: $A: 450=$ absorbância; $L=$ largura da cubeta em $\mathrm{cm}$; e $\mathrm{W}=$ quociente entre a massa da amostra $(\mathrm{g})$ e o volume final da diluição $(\mathrm{mL})$.

\subsubsection{Flavonoides Amarelos (mg.100 g-1)}

Determinado por espectrofotometria a $374 \mathrm{~nm}$, seguindo a metodologia de Francis (1982), utilizando $2 \mathrm{~g}$ de polpa para $10 \mathrm{~mL}$ da solução Etanol PA - $\mathrm{HCl}$ (85:15). Para cálculos foi utilizada a fórmula 2: Flavonóides Amarelos = fator de diluição $\mathrm{x}$ absorbância/76,6. 


\subsubsection{Antocianinas (mg.100 g-1)}

Determinada por espectrofotometria a $535 \mathrm{~nm}$, utilizando-se solução extratora de Etanol - HCL(85:15), seguindo metodologia descrita por Francis (1982). Os dados foram calculados através da fórmula 3: fator de diluição x absorbância/76,6.

\subsection{DETERMINAÇÃO DE ATIVIDADE ANTIOXIDANTE TOTAL (AAT) E POLIFENÓIS EXTRAÍVEIS TOTAIS (PET)}

\subsubsection{Preparo dos extratos fenólicos}

Os extratos fenólicos foram obtidos a partir de $3 \mathrm{~g}$ de cada amostra congelada em freezer a $-80^{\circ} \mathrm{C}$, pesados em tubos falcon e extraídos sequencialmente com $4 \mathrm{~mL}$ de metanol (50 \%) em temperatura ambiente por 1 hora para a extração. Os tubos foram centrifugados a $9.000 \mathrm{rpm}$, por 15 minutos, e o sobrenadante foi recolhido e colocando em tubo de ensaio graduado. Posteriormente, adicionou-se ao resíduo $4 \mathrm{~mL}$ de acetona (70\%), e os tubos foram mantidos a temperatura ambiente por 1 hora para a extração, após este período foram centrifugados a $9.000 \mathrm{rpm}$, por 15 minutos, sendo recolhido o sobrenadante. Após os sobrenadantes da extração com metanol e acetona foram combinados em provetas graduadas e adicionados de água destilada até completar o volume para $10 \mathrm{~mL}$. A extração foi realizada em triplicata, para cada planta seguindo metodologia descrita por Larrauri et al. (1997). Este extrato foi utilizado para determinar o conteúdo de polifenóis extraíveis totais e a atividade antioxidante.

\subsubsection{Determinação de polifenóis extraíveis totais}

O conteúdo de polifenóis extraíveis totais (PET) foi determinado pelo método de Folin-Ciocalteau, conforme metodologia citada por Larrauri et al. (1997), utilizando ácido gálico como padrão, e os resultados, expressos em mg.100 $\mathrm{g}^{-1}$ de polpa. Foi utilizado uma alíquota de $200 \mu \mathrm{L}$ dos extratos fenólicos combinados com $1 \mathrm{~mL}$ do reagente Folin-Ciocalteu, $2 \mathrm{~mL}$ carbonato de sódio $20 \%$ e 2,8 mL de água destilada, preparados em tubos de ensaio. Os tubos foram agitados e incubados durante 30 minutos a temperatura ambiente, todos os procedimentos realizados ao abrigo de luz. As medições de absorbância foram realizadas em espectrofotômetro no comprimento de onda a $700 \mathrm{~nm}$. Todas as determinações foram efetuadas em duplicata de cada repetição.

\subsubsection{Determinação da atividade antioxidante total}

A atividade antioxidante total (ATT) foi avaliada por meio da capacidade dos antioxidantes, presentes nas amostras, em sequestrar o radical livre ABTS •+, de acordo com metodologia descrita por Rufino et al. (2007). A partir do extrato fenólico, preparou-se em tubos de ensaio, três diluições ( $10 \mu \mathrm{L} ; 20 \mu \mathrm{L}$ e $30 \mu \mathrm{L}$ ) completando para $30 \mu \mathrm{L}$ com água destilada. Essa diluição foi acrescida de $3 \mathrm{~mL}$ do reagente radical ABTS •+. Agitou-se o tubo de ensaio, e após 6 minutos, todos os procedimentos realizados ao abrigo de luz. As medições de absorbância foram realizadas em espectrofotômetro no comprimento de onda a $734 \mathrm{~nm}$. 


\subsection{DELINEAMENTO EXPERIMENTAL E ANÁLISE ESTATÍSTICA}

O delineamento experimental utilizado foi o inteiramente casualizado. Os dados foram submetidos a análise de variância (ANOVA), com auxílio do programa Sisvar versão 5.1 (Ferreira, 2014). Quando detectada diferença significativa pelo teste $F$, as médias das plantas foram comparadas pelo teste de Tukey, a $5 \%$ de probabilidade de erro $(p \leq 0,05)$.

A análise multivariada, com base na análise de componentes principais, estudando a resposta aos fatores estudados para o autovalor da matriz de correlação $(\lambda)$ e a determinação da contribuição dos componentes bioativos com a atividade antioxidante das amostras avaliadas, foi realizada a correlação de Pearson e com auxílio do programa STATISTICA $7.0^{\circledR}$ (StatSoft, Tulsa, OK, USA).

\section{RESULTADOS E DISCUSSÃO}

Pelos resultados apresentados na Tabela 1, verificou-se diferenças significativas $(p \leq 0,05)$ entre os acessos quanto o conteúdo de ácido ascórbico, com variação de 45,20 a $55,80 \mathrm{mg} .100 \mathrm{~g}^{-1}$ (A3 e A1) e média de $46,49 \mathrm{mg} .100 \mathrm{~g}^{-1}$. Estes valores são semelhantes aos reportados por Kohatsu et al. (2011) com conteúdo de ácido ascórbico variando de 35,9 a 54,2 mg. $100 \mathrm{~g}^{-1}$ para este mesmo fruto. Por outro lado, são superiores aos citados por Barreto et al. (2009), 28,2 mg.100 g ácido ascórbico.

Esses resultados mostram que os frutos da cajá-mangueira apresentam conteúdo de ácido ascórbico superior ao de outras Spondias. Santos et al. (2010) e Silva et al. (2011) reportaram valores médios de 16,4 e 34,18 mg.100 g $\mathrm{g}^{-1}$, respectivamente, em frutos de umbucajazeira (Spondias sp.). Melo et al. (2010) e Rufino et al. (2010), reportaram valores médios de 9,38 e $8,0 \mathrm{mg} .100 \mathrm{~g}^{-1}$, respectivamente, para frutos de umbuzeiro (Spondiastuberosa Arruda) colhidos na maturidade comercial. Mas também superior ao conteúdo de ácido ascórbico de frutas usualmente consumidas, como por exemplo a laranja pêra e o abacaxi, com conteúdo médio de 37,34 e 24,98 mg.100 g ${ }^{-1}$, respectivamente (Melo et al., 2006).

O conteúdo de ácido ascórbico é um composto presente naturalmente nas frutas, visto como um parâmetro nutricional muito relevante, definido como um composto antioxidante presente nos vegetais que reduz a oxidação nas células devido a geração de radicais livres em excesso, ocasionados por doenças, desse modo o elevado conteúdo de ácido ascórbico presentes nos frutos da cajá-mangueira aumenta seu potencial para a comercialização devido ao potencial funcional de seus compostos (Sharma et al., 2008; Santos et al., 2010).

O conteúdo de carotenoides total nos diferentes acessos variou de 143,55 a 343,72 $\mu \mathrm{g} .100 \mathrm{~g}^{-1}$ (Tabela 1). A maior média encontrada foi para o acesso A2 $\left(343,72 \mu \mathrm{g} .100 \mathrm{~g}^{-1}\right)$, diferindo significativamente dos demais acessos. Barreto et al. (2009), avaliando frutos de 18 frutíferas tropicais adquiridas no Brasil, verificaram para esta mesma espécie um valor médio de $18,2 \mathrm{mg} \cdot 100 \mathrm{~g}^{-1}$.

Melo et al. (2010) e Rufino et al. (2010) reportaram conteúdo de carotenoides totais de $302,0 \mu \mathrm{g} .100 \mathrm{~g}^{1}$ e $10.0 \mu \mathrm{g} . \mathrm{g}^{1}$, respectivamente, para frutos maduros de umbuzeiro (Spondiastuberosa Arruda). Por outro lado, Tiburski et al. (2011) encontraram valores médios superiores com $48.69 \mu \mathrm{g} \cdot \mathrm{g}^{-1}$.

Quanto ao conteúdo de flavonoides amarelos houve diferença significativa $(p \leq 0,05)$ entre os acessos avaliados, os valores oscilaram entre 1,61 a $2,55 \mathrm{mg} 100 \mathrm{~g}^{-1}$, com média de $1,95 \mathrm{mg} \cdot 100 \mathrm{~g}^{-1}$ (Tabela 1). Estes valores são inferiores aos citados por Barreto et al. (2009), 
com $18,2 \mathrm{mg} \cdot 100 \mathrm{~g}^{-1}$ para este mesmo fruto. No entanto, são semelhantes aos resultados obtidos por Silva et al. (2013) para frutos de cajá (Spondias mombin L), com valores variando entre $1,87 \mathrm{mg} .100 \mathrm{~g}^{-1}$ e $5,64 \mathrm{mg} .100 \mathrm{~g}^{-1}$, com média geral de $3,22 \mathrm{mg} .100 \mathrm{~g}^{-1}$, sendo ligeiramente inferior aos reportados por Rufino et al. (2010), para frutos de cajá (Spondias mombin $\mathrm{L}$ ) e umbu com valores de 7,1 e $6,9 \mathrm{mg} .100 \mathrm{~g}^{-1}$, respectivamente, ambas pertencentes ao gênero Spondias.

O conteúdo de antocianinas diferiu significativamente $(p \leq 0,05)$ entre os acessos avaliados, variando de 0,36 a $0,49 \mathrm{mg} \cdot 100 \mathrm{~g}^{-1}$, cuja média foi de $0,43 \mathrm{mg} \cdot 100 \mathrm{~g}^{-1}$ (Tabela 1). Destacando-se o acesso A3 que apresentou o maior conteúdo com 0,49 mg.100 g ${ }^{-1}$. Valores próximos ao desta pesquisa foram citados por Rufino et al. (2010), 0,30 mg.100 g $\mathrm{g}^{-1} \mathrm{de}$ antocianinas, em frutos de umbuzeiro, provenientes de diferentes estados do Brasil.

Os níveis de metabólitos das plantas, dentre eles os carotenoides, flavonoides e antocianinas, apresentam variações em seus conteúdos, o que pode ser explicado por fatores genéticos (espécie de planta) e ambientais (temperatura, luminosidade, umidade, tipo de solo) (Kalt, 2005; Wang et al.,2009).

TABELA 1 - VALORES MÉDIOS DE ÁCIDO ASCÓRBICO, CAROTENOIDES TOTAIS, FLAVONOIDES AMARELOS E ANTOCIANINAS, DE FRUTOS DE DIFERENTES ACESSOS DE CAJÁ-MANGUEIRA (Spondiasdulcis Parkinson) COLHIDOS MADUROS

\begin{tabular}{|c|c|c|c|c|}
\hline Acesso & $\begin{array}{l}\text { Ácido Ascórbico } \\
\left(\mathrm{mg} .100 \mathrm{~g}^{-1}\right)\end{array}$ & $\begin{array}{l}\text { Carotenoides totais } \\
\quad\left(\mu \mathrm{g} .100 \mathrm{~g}^{-1}\right)\end{array}$ & $\begin{array}{c}\text { Flavonoides } \\
\text { amarelos }_{1} \text { (mg.100 }\end{array}$ & $\begin{array}{l}\text { Antocianinas } \\
\left(\mathrm{mg}^{\prime} 100 \mathrm{~g}^{-1}\right)\end{array}$ \\
\hline $\mathrm{A} 1$ & $55,80 \mathrm{~A}$ & $196,34 \mathrm{~B}$ & $1,61 \mathrm{~B}$ & $0,36 \mathrm{~B}$ \\
\hline A2 & $38,49 \mathrm{C}$ & $343,72 \mathrm{~A}$ & $2,55 \mathrm{~A}$ & $0,45 \mathrm{AB}$ \\
\hline A3 & $45,20 \mathrm{~B}$ & $143,55 \mathrm{~B}$ & $1,68 \mathrm{~B}$ & $0,49 \mathrm{~A}$ \\
\hline Mínimo & 37,61 & 118,73 & 1,45 & 0,30 \\
\hline Máximo & 57,71 & 416,77 & 2,63 & 0,53 \\
\hline Média & 46,49 & 227,87 & 1,95 & 0,43 \\
\hline
\end{tabular}

*Valores seguidos de mesma letra na coluna não diferem estatisticamente entre si pelo Teste de Tukey, em nível de significância de $5 \%$.

Os valores médios polifenóis extraíveis totais (PET) oscilaram de 46,19 a 55,38 mg.100 $\mathrm{g}^{-1}$, não diferindo significativamente $(p \leq 0,05)$ entre os acessos avaliados (Tabela 2$)$. Estes valores, no entanto, foram inferiores aos citados por Barreto et al. (2009), 99,4 mg.100 g ${ }^{1}$ para este mesmo fruto colhido na maturidade comercial.

Com base na classificação proposta por Vasco et al. (2008), pode-se classificar os polifenóisem baixo $\left(<1 \mathrm{mg} .100 \mathrm{~g}^{-1}\right)$, médio $\left(1-5 \mathrm{mg} .100 \mathrm{~g}^{-1}\right)$ e alto $\left(>5 \mathrm{mg} .100 \mathrm{~g}^{-1}\right)$ teor. De acordo com esta classificação, pode-se inferir que os frutos de cajá-mangueira estudados neste trabalho apresentam elevada concentração de fenóis.

Comparando o conteúdo de PET de cajá-manga com outros frutos do mesmo gênero botânico, o teor de polifenóis foi inferior aos citados por Silva et al. (2013), 60,47 mg $100 \mathrm{~g} \mathrm{~g}^{-1}$, Vieira et al. (2011), 70,92 mg.100 g $\mathrm{g}^{-1}$, Tiburski et al. (2011), 260,21 mg.100 g $\mathrm{g}^{-1}$ e por Rufino et al. (2010), 72,00 mg.100 g ${ }^{-1}$ para frutos de cajazeira (Spondias mombin L.). Por outro lado, para frutos de umbuzeiro (Spondiastuberosa Arr. Cam.) Dantas Júnior (2008) e Almeida et al.

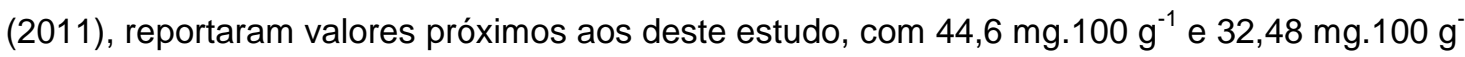
', respectivamente, já Genovese et al. (2008), citaram valor inferior, com 34,0 mg.100 g ${ }^{-1}$. 
Dentre os compostos fenólicos, estão presentes os ácidos fenólicos, cumarinas, flavonoides, taninos condensados e ligninas. Estes compostos nos vegetais atuam na pigmentação, proteção contra a luz UV (Kuskoski et al., 2005; Santos et al., 2008). De acordo com Prior et al. (1998) e Niki et al. (2005) estes compostos são os principais responsáveis pela atividade antioxidante, que apresenta ação redutora de espécies reativas de oxigênio e nitrogênio, que quando em excesso, podem causar danos celulares e contribuir para o surgimento de doenças.

Os valores médios para a atividade antioxidante total não diferiu significativamente entre os acessos avaliados ( $p \leq 0,05)$, com valores variando de 5,14 a 6,37 $\mu \mathrm{M}$.trolox $\mathrm{g}^{-1}$, cuja média foi de 5,87 $\mu \mathrm{M}$.trolox $\mathrm{g}^{-1}$ (Tabela 2). Estes resultados são superiores aos citados por Franquin et al. (2005) e Barreto et al. (2009), que reportaram valores médios de AAT por ABTS variando entre 0,50 e 2,10 $\mu \mathrm{M}$.trolox $\mathrm{g}^{-1}$ para este mesmo fruto no estádio de maturação maduro.

Comparando a AAT do cajá-manga com outras espécies do gênero Spondia, Almeida et al. (2011) e Gondim (2012) reportam valores inferiores ao deste estudo (0,31 e 1,07 $\mu \mathrm{M}$.trolox $\mathrm{g}^{-1}$ ) em frutos de umbu, (Spondias tuberosa Arr. Cam.) colhidos maduros, proveniente do nordeste brasileiro. No entanto, Almeida et al. (2011) e Rufino et al. (2010) reportaram valores superiores aos encontrados neste trabalho, com 6,25 $\mu \mathrm{M}$.trolox $\mathrm{g}^{-1}$ para ciriguela (Spondias purpurea L.) e 40,7 $\mu \mathrm{M}$.trolox $\mathrm{g}^{-1}$ para cajá (Spondias mombin L.), respectivamente.

A atividade antioxidante de cajá-manga, foi inferior à da polpa do açaí $(10,21$ a 52,47 $\mu \mathrm{M}$ de Trolox/g) e superior à da polpa de cupuaçu (1,11 a 1,57 $\mu \mathrm{M}$ de Trolox/g) (Kuskoski et al., 2006; Santos, 2007).

De acordo com Leong e Shui (2002), dentre os compostos com ação antioxidante, destacam-se os compostos bioativos, polifenóis e os carotenoides, em que as frutas exóticas e não tradicionais do nordeste brasileiro, são tidas como fonte promissora destes compostos. A quantidade destes compostos varia em função da espécie frutífera, condições edafoclimáticas da região de cultivo, estádio de maturação de colheita e métodos de processamento e armazenamento.

TABELA 2 - VALORES MÉDIOS DE POLIFENÓIS EXTRAÍVEIS TOTAIS (PET) E ATIVIDADE ANTIOXIDANTE TOTAL (AAT), DE FRUTOS DE DIFERENTES ACESSOS DA CAJÁMANGUEIRA (Spondiasdulcis Parkinson) COLHIDOS MADUROS

\begin{tabular}{ccc}
\hline Acesso & $\begin{array}{c}\text { PET } \\
\left(\mathrm{mg}^{\left.100 \mathrm{~g}^{-1}\right)}\right.\end{array}$ & $\begin{array}{c}\text { AAT } \\
\left(\mu \mathrm{M} . \text { trolox }^{-1}\right)\end{array}$ \\
\hline A1 & $46,19 \mathrm{~A}$ & $6,37 \mathrm{~A}$ \\
$\mathrm{~A} 2$ & $52,22 \mathrm{~A}$ & $6,12 \mathrm{~A}$ \\
$\mathrm{~A} 3$ & $55,38 \mathrm{~A}$ & $5,14 \mathrm{~A}$ \\
\hline Mínimo & 43,20 & 4,53 \\
\hline Máximo & 63,65 & 7,60 \\
\hline Média & 51,27 & 5,87 \\
\hline "Valores seguidos de mesma letra na coluna não diferem estatisticamente entre si pelo Teste de Tukey, em nível de \\
significância de 5\%.
\end{tabular}

A Tabela 3 apresenta as correlações entre os fatores compostos bioativos, polifenóis extraíveis totais e atividade antioxidante. A correlação fornece informações relevantes sobre a dinâmica entre as variáveis que validam os resultados. 
Houve correlação positiva, em nível de $1 \%$, entre a atividade antioxidante total, com o teor ácido ascórbico (Tabela 3). Para os compostos bioativos verificaram-se correlações positivas, em nível de $1 \%$, entre os flavonoides amarelos totais $\left(0.81^{* *}\right)$, com os carotenoides totais. Foram observadas correlações negativas significativas, em nível de $1 \%$, entre os flavonoides amarelos totais $\left(-0,80^{* \star}\right)$ com o teor de carotenoides totais.

A correlação positiva também foi observada por Silva et al., (2012), avaliando a correlação entre os compostos bioativos, os polifenóis extraíveis total e a atividade antioxidante em cajá (Spondias mombin L), fruto do mesmo gênero botânico, reportaram correlação significativa entre os flavonoides amarelos $\left(0,64^{* *}\right)$, com os carotenoides totais. Estes dados corroboram com Dudonné et al. (2009), ao afirmarem que a presença de compostos fenólicos em extratos de vegetais apresenta correlação significativa como potencial antioxidante.

TABELA 3 - CORRELAÇÕES DE PERSON ENTRE OS COMPOSTOS BIOATIVOS, OS POLIFENÓIS EXTRAÍVEIS TOTAIS E A ATIVIDADE ANTIOXIDANTE TOTAL, DE FRUTOS DE DIFERENTES ACESSOS DA CAJÁ-MANGUEIRA (Spondiasdulcis Parkinson) COLHIDOS MADUROS

\begin{tabular}{ccccccc}
\hline Variáveis & Ác. Ascórbico & Carotenoides & Flav. Amarelos & Antocianinas & PET & AAT \\
\hline Ác. Ascórbico & 1,00 & $-0,59$ & $-0,80^{* *}$ & $-0,54$ & $-0,46$ & $0,84^{*}$ \\
Carotenoides & - & 1,00 & $0,81^{* *}$ & $-0,16$ & $-0,03$ & 0,09 \\
Flav. Amarelos & - & - & 1,00 & 0,26 & 0,02 & 0,21 \\
Antocianinas & - & - & - & 1,00 & 0,38 & 0,07 \\
PET & - & - & - & - & 1,00 & $-0,04$ \\
AAT & - & - & - & - & - & 1,00 \\
\hline
\end{tabular}

$\left({ }^{* *}\right)$ e $\left({ }^{*}\right)$ significativo a 1 e $5 \%$ de probabilidade, respectivamente, pelo teste t.

A análise de componentes principais foi utilizada para resumir os compostos bioativos e a atividade antioxidante permitindo identificar três componentes principais, que representaram 87,33 \% da variância total das características originais (Tabela 4).

Avaliando os autovetores na Tabela 4, verifica-se que todos os compostos bioativos e a atividade antioxidante avaliados nos frutos de cajá-mangueira, foram importantes para explicar de forma satisfatória a variabilidade ocorrida entre os frutos dos acessos de cajámangueira colhidos maduros. Na Figura 1, observa-se a dispersão gráfica dos compostos bioativos, atividade antioxidante e os acessos de cajá-mangueira avaliados. Estes resultados são semelhantes aos citados por Dudonné et al. (2009), que ao determinarem o total de conteúdo fenólico e caracterizarem a atividade antioxidante de trinta plantas por diferentes medotos, dentre eles ABTS, concluíram que a presença de compostos fenólicos em extratos de vegetais apresenta alta correlação positiva com a capacidade antioxidante.

Sendo o conteúdo de flavonoides amarelos, carotenoides totais e ácido ascórbico, por ordem de importância, relacionados ao componente principal 1, respondendo por $44,23 \%$ da variabilidade. Para a componente principal (CP2) verificou-se que o conteúdo de polifenóis extraíveis totais respondendo a $25,58 \%$ da variabilidade (Tabela 4, Figura 1), indicando que estes compostos bioativos contribuem para atividade antioxidante do cajá-manga. Enquanto que, a atividade antioxidante responde a $17,52 \%$ da variabilidade no componente principal 3 .

A correlação entre os compostos bioativos e a tividade antioxidante também foi verificada por Gondim (2012) e Silva et al., (2012) que reportaram correlação significativa entre os conteúdos de carotenoides e polifenóis extraíveis totais, com a capacidade antioxidante pelo 
método ABTS, em frutos de umbuzeiro (Spondias tuberosa Arr. Cam.) e cajazeira (Spondias mombin L.), respectivamente.

Desse modo, nota-se que a atividade antioxidante total em cajá manga, determinada pela captura do radical $\mathrm{ABTS}{ }^{\bullet+}$ está sendo expressada principalmente pelo conteúdo de ácido ascórbico da polpa.

Tabela 4 - AUTOVETORES EM TRÊS COMPONENTES PRINCIPAIS (CP1, CP2 E CP3), DOS COMPOSTOS BIOATIVOS E DA ATIVIDADE ANTIOXIDANTE DE FRUTOS DE ACESSOS DE CAJÁ-MANGUEIRA (Spondiasdulcis Parkinson) COLHIDOS MADUROS

\begin{tabular}{cccc}
\hline Variáveis & CP1 & CP2 & CP3 \\
\hline Acido Ascórbico & $-0,952756^{*}$ & $-0,200296$ & $-0,183501$ \\
Carotenoides totais & $0,740649^{*}$ & $-0,591989$ & 0,158239 \\
Flavonoides amarelos & $0,915176^{*}$ & $-0,337208$ & $-0,074211$ \\
Antocianinas & 0,467265 & 0,686915 & $-0,297762$ \\
Polifenóis extraíveis totais & 0,365446 & $0,705082^{*}$ & 0,001691 \\
Atividade antioxidante total & 0,090310 & $-0,248187$ & $-0,947841^{*}$ \\
\hline$\lambda$ & 2,65 & 1,53 & 1,05 \\
Variância acumulada (\%) & 44,23 & 25,58 & 17,52 \\
\hline
\end{tabular}

FIGURA 1- DISPERSÃO GRÁFICA DA ANÁLISE DAS COMPONENTES PRINCIPAIS DOS COMPOSTOS BIOATIVOS E ATIVIDADE ANTIOXIDANTE, EM FRUTOS DE ACESSOS DE CAJÁ-MANGUEIRA (Spondiasdulcis Parkinson) COLHIDOS MADUROS

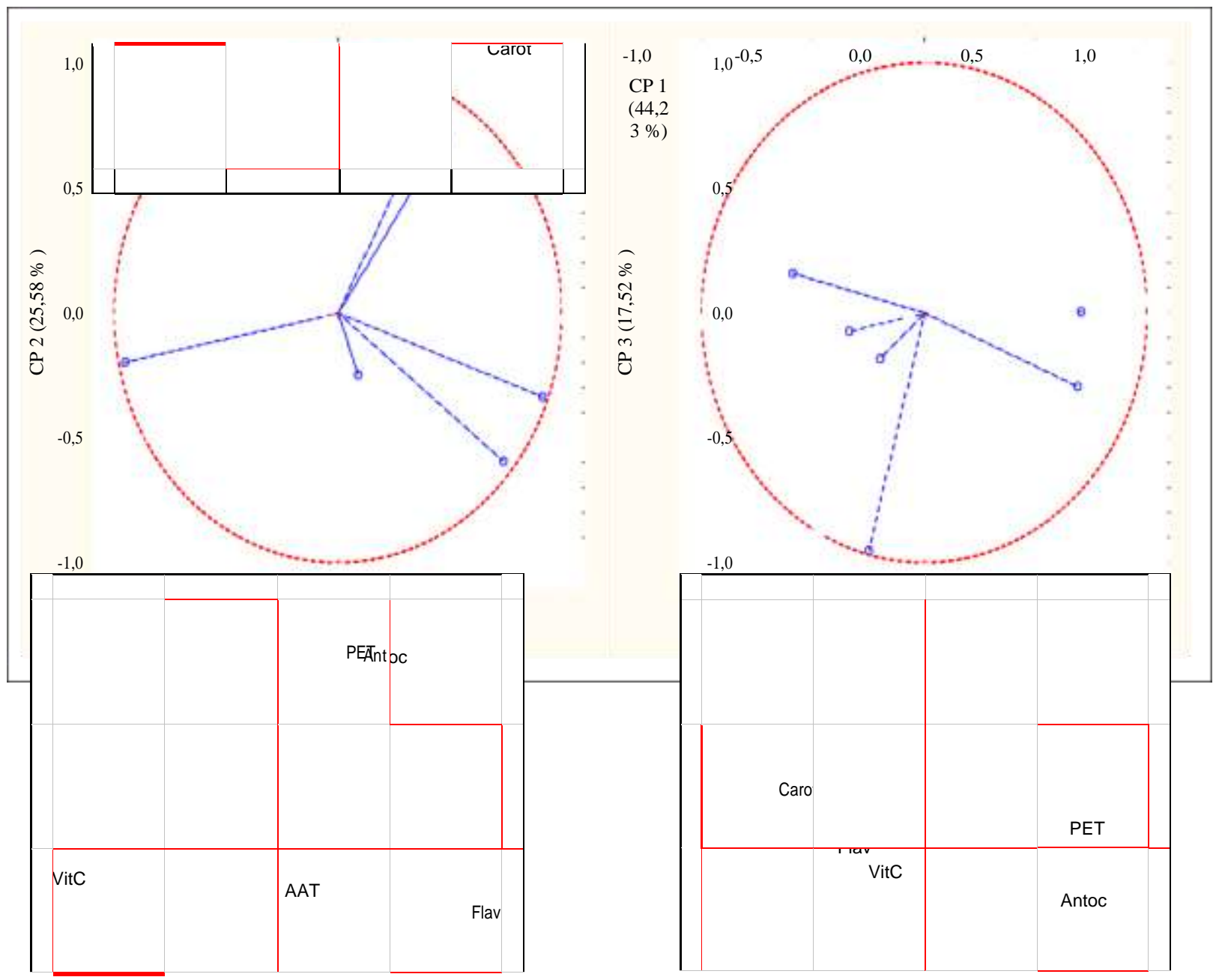




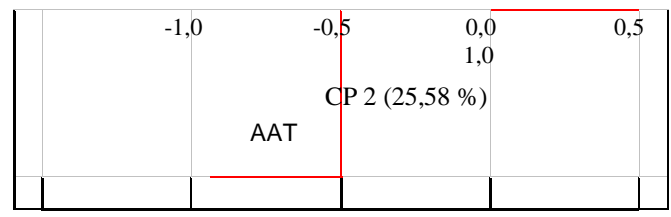

\section{CONCLUSÕES}

Considerando os baixos teores de flavonoides amarelos e antocianinas em polpa de cajá-manga, é possível inferir que a ação antioxidante exibida decorra, principalmente, da presença de ácido ascórbico e polifenóis extraíveis totais. 
O conteúdo de ácido ascórbico do cajá-manga foi superior ao conteúdo de ácido ascórbico de frutas usualmente consumidas, como por exemplo a laranja pêra e o abacaxi. A atividade antioxidante do cajá-manga é próxima ao de frutas com um potencial antioxidante conhecido (cupuaçu), o que permite inferir que este fruto é uma fonte potencial de antioxidantes para a dieta humana.

O conteúdo de flavonoides amarelos apresentou forte correlação com o de carotenoides e a atividade antioxidante foi fortemente correlacionada com o conteúdo de ácido ascórbico.

O conteúdo de ácido ascórbico, carotenoides totais, flavonoides amarelos, antocianinas, polifenóis extraíveis totais e atividade antioxidante contribuirão para a variabilidade entre acessos, com ácido ascórbico inferindo a maior contribuição para esta variação.

\section{AGRADECIMENTOS}

Ao Conselho Nacional de Desenvolvimento Científico e Tecnológico (CNPq) pelo auxílio financeiro e pela concessão da bolsa de iniciação cientifica ao primeiro autor.

\section{ABSTRACT \\ PHENOLIC COMPOUNDS, CAROTENOIDS AND ACTIVITY ANTIOXIDANT IN CAJA-MANGO FRUITS}

The present work had as objective to quantify the phenolic compounds, carotenoids and antioxidant activity of cajá-mangueira fruits of different genotypes of spontaneous occurrence in the State of Paraíba. The experimental design was the completely randomized (DIC), with three accesses (A1, A2 and A3), in three replicates. The fruits were analyzed for ascorbic acid content, total carotenoids, yellow flavonoids, anthocyanins, total extractable polyphenols and antioxidant activity. The genotypes differed significantly as to the content of ascorbic acid, total carotenoids, yellow flavonoids and anthocyanins. The results showed that cajá-manga has significant amounts of ascorbic acid $\left(46,49 \mathrm{mg} \cdot 100 \mathrm{~g} \mathrm{~g}^{-1}\right)$. Considering the low levels of yellow flavonoids and anthocyanins in cajá-mangueira pulp, it is possible to infer that the antioxidant action shown is mainly due to the presence of ascorbic acid and total extractable polyphenols. Ascorbic acid content was higher than fruit usually consumed and antioxidant activity close to fruit with a known antioxidant potential. The antioxidant activity was strongly correlated with ascorbic acid content. The content of ascorbic acid, total carotenoids, yellow flavonoids and anthocyanins will contribute to the variability among accesses, with ascorbic acid inferring the greater contribution to this variation.

KEY-WORDS: POSTHARVEST; TROPICAL FRUITS; ANACARDIACEAE; SPONDIASDULCIS PARKINSON; ASCORBIC ACID; ABTS

\section{REFERÊNCIAS}

1 ALI, S.S.; KASOJU, N.; LUTHRA, A.; SINGH, A.; SHARANABASAVA, H., SAHU, A.; BORA, U. Indian medicinal herbs as sources of antioxidants. Food Research International, v.41, n.1, p.1-15, 2008.https://doi.org/10.1016/j.foodres.2007.10.001 
2 ALMEIDA, M.M.B.; SOUSA, P.H.M.; ARRIAG, A.M. C.; PRADO, G.M.; MAGALHÃES, C.E.C.; MAIA, G.A.; LEMOS, T.L.G. Bioactive compounds and antioxidant activity of fresh exotic fruits from northeastern Brazil. Food Research Internatinal, v.44, n.7, p.2155-2159, 2011. https://doi.org/10.1016/j.foodres.2011.03.051

3 AROUCHA, E.M.M.; SOUZA, C.S.M.; SOUZA, A.E.D.; FERREIRA, R.M.A. AROUCHA FILHO, J.C. Qualidade pós-colheita da cajarana em diferentes estádios de maturação durante armazenamento refrigerado. Revista Brasileira de Fruticultura, v.34, n.2, p.391-399, 2012. http://dx.doi.org/10.1590/S0100-29452012000200011

4 BARRETO, G.P.M.;BENASSIB, M.T.; MERCADANTE, A.Z. Bioactive Compounds from Several Tropical Fruits and Correlation by Multivariate Analysis to Free Radical Scavenger Activity. Journal of the Brazilian Chemical Society, v.20, n.10, p.1856-1861, 2009. http://dx.doi.org/10.1590/S010029452012000200011

5 CARVALHO, P.C.L.; RITZINGER, R.; SOARES FILHO, W.S.; LEDO, C.A.S. Características morfológicas, físicas e químicas de frutos de populações de umbu-cajazeira no Estado da Bahia. Revista Brasileira de Fruticultura, v.1, n.30, p.140-147, 2008. http://dx.doi.org/10.1590/S010029452008000100026

6 DANTAS JUNIOR, O.R.D. Qualidade e capacidade antioxidante total de frutos de genótipos de umbuzeiro oriundos do semi-árido nordestino. 2008. 106 f. Tese (Doutorado em Agronomia) Universidade Federal da Paraíba, Areia, 2008.

7 DANTAS, A.L., SILVA, S.M., DANTAS, R.L., MENDONÇA, R.M.N., SANTOS, L.F., SANTOS, L.S. Mudanças nos compostos bioativos em frutos da cajá-mangueira (Spondias cytherea sonn.) na maturação. Anais: III SIMPÓSIO BRASILEIRO DE PÓS-COLHEITA - SPC 2011, Nova Friburgo-RJ (publicado em CD-ROM).

8 DUDONNÉ, S.; VITRAC, X.; COUTIERE, P.; WOILLEZ, M.; MÉRILON, J.M. Comparative Study of Antioxidant Properties and Total Phenolic Content of 30 Plant Extracts of Industrial Interest Using DPPH, ABTS, FRAP, SOD, and ORAC Assays. Journal of Agricultural and Food Chemistry, v.57, n.5, p.1768-1774, 2009. http://dx.doi.org/10.1021/jf803011r

9 FERREIRA, D.F. Sisvar: A Guide for its Bootstrap procedures in multiple comparisons. Ciência \& Agrotecnologia, v.38, n.2, p.109-112, 2014. http://dx.doi.org/10.1590/S1413-70542014000200001

10 FRANCIS, F.J. Analysis of anthocyanins. In: MARKAKIS, P. (ed.). Anthocyanins as food colors. New York: Academic Press, p.181-207, 1982.

11 FRANQUIN, S.; MARCELIN, O.; AURORE, G.; REYNES, M.; BRILLQUET, J.M. Physicochemical characterisation of the mature-green Golden apple (Spondias cytherea Sonnerat). Fruits, v.60, n.3, p.203-210, 2005. http://dx.doi.org/10.1051/fruits:2005027

12 GENOVESE, M.I.; PINTO, M.S.; GONÇALVES, A.E.S.S.; LAJOLO, F.M. Bioactive Compounds and Antioxidant Capacity of Exotic Fruits and Commercial Frozen Pulps from Brazil. Food Science and Technology International, v.14, n.3, p.207-214, 2008. https://doi.org/10.1177/1082013208092151

13 GONÇALVES, A.E.S.S.; LAJOLO, F.M.; GENOVESE, M.I. Chemical Composition and Antioxidant/Antidiabetic Potential of Brazilian Native Fruits and Commercial Frozen Pulps. Journal of Agricultural Food Chemistry, v.58, n.8, p.4666-4674, 2010. https://doi.org/10.1021/jf903875u

14 GONDIM, P.J.S. Identificação de carotenoides e quantificação de compostos bioativos e atividade antioxidante em frutos do gênero Spondias. 2012. $104 \mathrm{f}$. Tese (Doutorado em Agronomia) - Universidade Federal da Paraíba. Areia, 2012.

15 HIGBY, W.K. A simplified method for determination of some the carotenoid distribuition in natural and carotene-fortified orange juice. Journal of Food Science, v.27, n.1, p.42-49, 1962. https://doi.org/10.1111/j.1365-2621.1962.tb00055.x

16 JANICK, J.; PAULL, R.E. The encyclopedia of fruit \& nuts. Wallingford, UK: CABI International Publishing, 2008. $954 \mathrm{p}$.

17 KALT, W. Effects of production and processing factors on major fruit and vegetable antioxidants. Journal of Food Science, v.70, n.1, p.11-19, 2005. https://doi.org/10.1111/j.13652621.2005.tb09053.x 
18 KOHATSU, D.S.; ZUCARELI, V.; BRAMBILLA, W.P.; EVANGELISTA, R.M. Qualidade de frutos de cajá-manga armazenados sob diferentes temperaturas.Revista Brasileira de Fruticultura, v.33, n.spe, p.344-349, 2011. http://dx.doi.org/10.1590/S0100-29452011000500043

19 KUSKOSKI, E.M.; ASUERO, A.G.; MORALES, M.T.; FETT, R. Frutos tropicais silvestres e polpas de frutas congeladas: atividade antioxidante, polifenóis e antocianinas. Ciência Rural, v.36, n.4, p.1283-1287, 2006. http://dx.doi.org/10.1590/S0103-84782006000400037

20 KUSKOSKI, E.M.; ASUERO, G.A.; TRONCOSO, A.M.; MANCINIFILHO, J.; FETT, R. Aplicación de diversos métodos químicos para determinar actividad antioxidante em pulpa de frutos. Ciência e Tecnologia de Alimentos, v.25, n.4, p.726-732, 2005. http://dx.doi.org/10.1590/S010120612005000400016

21 LARRAURI, J.A.; RUPÉREZ, P.; SAURA-CALIXTO, F. Effect of drying temperature on the stability of polyphenols and antioxidant activity of red grape pomace peels. Journal of Agricultural and Food Chemistry,v.45, n.4, p.1390-1393, 1997. https://doi.org/10.1021/jf960282f

22 LEONG, L.P.; SHUI, G. An investigation of antioxidant capacity of fruit in Singapore markets. Food Chemistry, v.76, n.1, p.69-75, 2002. https://doi.org/10.1016/S0308-8146(01)00251-5

23 LORENZI, H. Frutas Brasileiras e exóticas cultivadas (de consumo in natura). São Paulo: Instituto plantarum de estudos da Flora, 2006, 672p.

24 LU, M.; YUAN, B.; ZENG, M.; CHEN, J. Antioxidant capacity and major phenolic compounds of spices commonly consumed in China. Food Research International, v.44, n.2, p.530-536. 2011. http://dx.doi.org/10.1016/j.foodres.2010.10.055

25 MELO, E.A.; ANDRADE, R.M.S. Compostos Bioativos e Potencial Antioxidante de frutos do umbuzeiro. Alimentos e Nutrição Araraquara, v.21, n.3, p.453-457, 2010.

26 MELO, E.A.; LIMA V.L.A.G.; MACIEL, M.I.S. Polyphenol, ascorbic ascid and total carotenoid contents in common fruits and vegetables. Brazilian Jornaul of Food Technology, v.9, n.2, p.89-94, 2006.

27 MOREIRA, A.C.C.G. Caracterização de frutos de genótipos de cajá-umbuzeiras: teor de fitoquímicos bioativos e potencial antioxidante. 122p, 2011. Dissertação (Mestrado em Ciência e Tecnologia de Alimentos) - Universidade Federal de Pernambuco, Recife.

28 MOURA, F.T.; SILVA, S.M.; SCHUNEMANN, A.P.P.; MARTINS, L.P. Frutos do umbuzeiro armazenados em diferentes estádios de maturação. Revista Ciência Agronômica, v.44, n.4, p.131133, 2013. http://dx.doi.org/10.1590/S1806-66902013000400013

29 NIKI, E.; YOSHIDA, Y.; SAITO, Y.; NOGUCHI, N. Lipid peroxidation: Mechanisms, inhibition, and biological effects. Biochemical and Biophysical Research Communications, v.338, n.1, p.668-676, 2005. https://doi.org/10.1016/j.bbrc.2005.08.072

30 PALIYATH, G.; MURR, D.P. Biochemistry of Fruits. In: PALIYATH, G.; MURR, D.P.; HANDA, A.K.; LURIE, S. (eds) Postharvest Biology and Technology of Fruits, Vegetables and Flowers. WileyBlackwell Publishing, cap 3, p. 19-50, 2008.

31 PRIOR, R.L.; CAO, G.; MARTIN, A.; LISCHNER, N.; EHLENFELDT, M.; KALT, W.; KREWER, G.; MAINLAND, C.M. Antioxidant capacity as influenced by total phenolics and anthocyanin content, maturity, and variety of Vaccinum species. Journal of Agricultural and Food Chemistry, v.46, n.7, p.2686-2693, 1998. https://doi.org/10.1021/jf980145d

32 RUFINO, M.S.M.; ALVES, R.E.; BRITO, E.S.; MORAIS, S.M.; SAMPAIO, C.G.; PÉREZ-JIMENEZ, J.; SAURA-CALIXTO, F.D. Metodologia científica: determinação da atividade antioxidante total em frutas pela captura do radical livre ABTS•+. Fortaleza: Embrapa Agroindústria Tropical, 2007. 4f. (Embrapa Agroindústria Tropical, Comunicado Técnico, 128).

33 RUFINO, M.S.M.; ALVES, R.E.; BRITO, E.S.; PÉREZ-JIMÉNEZ, J.; SAURA-CALIXTO, F.; MANCINIFILHO, J. Bioactive compounds and antioxidant capacities of 18 non-traditional tropical fruits from Brazil. Food Chemistry, v.121, n.4, p.996-1002, 2010. https://doi.org/10.1016/j.foodchem.2010.01.037

34 SANTOS, G.M. Contribuição da vitamina C, carotenóides e compostos fenólicos no potencial antioxidante de produtos comerciais de açaí e cupuaçu. 97p, 2007. Dissertação (Mestrado em Tecnologia de Alimentos) - Universidade Federal do Ceará, Fortaleza. 
35 SANTOS, G.M.; MAIA, G.A.; SOUSA, P.H.M; COSTA, J.M.C. FIGUEIREDO, R.W.; PRADO, G.M. Correlação entre atividade antioxidante e compostos bioativos de polpas comerciais de açaí (Euterpe oleracea Mart). Archivos Latinoamericanos de Nutricion, v.58, n.2, p.187-192, 2008.

36 SANTOS, M.B.; CARDOSO, R.L.; FONSECA, A.A.O.; CONCEIÇÃO, M.N. Caracterização e qualidade de frutos de umbu-cajá (Spondias tuberosa x S. mombin) provenientes Do Recôncavo sul da Bahia. Revista Brasileira de Fruticultura, v.32, n.4, p.1089-1097, 2010. http://dx.doi.org/10.1590/S010029452011005000015

37 SHARMA, M.; SITBON, C.; SUBRAMANIAN, J.; PALIYATHIN, G. Changes in nutritional quality of fruits and vegetables during storage. In: PALIYATH, G.; MURR, D.P.; HANDA, A.K.; LURIE, S. (eds) Postharvest Biology and Technology of Fruits, Vegetables and Flowers. Wiley-Blackwell Publishing. 443-466. 2008

38 SILVA, F.V.G.; SILVA, S.M.; SILVA, G.; SCHUNEMANN, A.P. Quality and antioxidante activity during ripening of fruits from yellow mombin (Spondias mombin L.) genotypes. Actahorticulturae, v.1012, n.1, p.843-848, 2013. https://doi.org/10.17660/ActaHortic.2013.1012.114

39 SILVA, F.V.G.; SILVA, S.M.; SILVA, G.C.; MENDONÇA, R.M.N.; ALVES, R.E.; DANTAS, A.L. Bioactive compounds and antioxidant activity in fruits of clone and ungrafted genotypes of yellow mombin tree. Ciência Tecnologia de Alimentos, v.32, n.4, p.685-691, 2012. https://doi.org/10.1590/S0101-20612012005000101

40 SILVA, L.R.; ALVES, R.E.; ARAGÃO, F.A.Z.; SILVA, S.M.; MAIA, L.K.; NOGUEIRA, D.H. Qualidade de frutos de genótipos de umbu-cajazeiras (Spondias sp.) oriundos da microrregião de Iguatu-CE. Scientia Plena, v.7, n.8, p.1-7, 2011. https://doi.org/10.3895/rbta.v9n1.1699

41 SOUZA, F.X.; SOUSA, F.H.L.; MELO, F.I.O. Aspectos morfológicos de endocarpos de cajarana (Spondias cytherea Sonn. - Anacardiaceae). Revista Brasileira de Sementes,v.20, n.2, p.141-146, 1998. https://doi.org/10.17801/0101-3122/rbs.v20n2p379-384

42 STROHECKER, R.; HENNING, H.M. Analisis de vitaminas: métodos comprobados. Madrid: PAZ MONTALVO, 1967, 428p.

43 TIBURSKI, J.H.; ROSENTHAL, A.; DELIZA, R.; GODOY, R.L.O.; PACHECO, S. Nutritional properties of yellow mombin (Spondias mombin L.) pulp. Food Rosearch International, v.44, n.7, p.2326-2331, 2011. https://doi.org/10.1016/j.foodres.2011.03.037

44 VASCO, C.; RUALES, J.; KAMAL-ELDIN, A. Total phenolic compounds and antioxidant capacities of major fruits from Ecuador. Food Chemistry, v.111, n.4, p.816-823, 2008. https://doi.org/10.1016/j.foodchem.2008.04.054

45 VIEIRA, L.M.; SOUSA, M.S.B.; MANCINI-FILHO, J.; LIMA, A. Fenólicos totais e capacidade antioxidante in vitro de polpas de frutos tropicais. Revista Brasileira de Fruticultura, v.33, n.3, p.888897, 2011. http://dx.doi.org/10.1590/S0100-29452011005000099

46 WANG, S.Y.; CHEN, C.; WANG, C.Y. The influence of light and maturity on fruit quality and flavonoid content of red raspberries. Food Chemistry, v.112, n.3, p.676-684, 2009. http://dx.doi.org/10.1016/j.foodchem.2008.06.032 